\title{
Bemerkungen über den Vokalismus der ersten Silbe von tschuwaschischen Lehnwörtern in einigen finnisch-ugrischen Sprachen
}

Ein Forscher der Lautgestalt von tschuw. Lehnwörtern im Wotjakischen und Tscheremissischen sieht sich vor folgende ungeklärte Frage gestellt: was für einen phonetischen Unterschied gab es ursprünglich zwischen ur- bzw. alttürk. ? ( $>$ tschuw. $\hat{\theta}$, a, s. MARTTI RÄsänen Zur Lautgeschichte der türkischen Sprachen 66, F. G. Is-chakov im Werke Issledovanije po sravnitel'noj grammatike tjurkskich jazykov I 94 -95) und dem Laut, der im heutigen Tschuwaschischen durch den mittleren illabialen Vollvokal $i$ vertreten wird? Ein dem tschuw. $i$ ähnlicher Laut existiert auch im Türkmenischen, Jakutischen und Tuvinischen (= Sojotischen), obgleich es wenige gemeinsame Wörter zu geben scheint, die auch nur in zwei der erwähnten Sprachen die $i$-Vertretung aufweisen. Doch erklärt sich nach GY. NÉmETH das Vorkommen von $i$ in denselben tschuw. und jakut. Wörtern aus der ehemaligen Einheit dieser Sprachen, s. NyK XLIII 290. Weitverbreitet ist wohl die z.B. von Räsänen vertretene Auffassung, dass sich sowohl tschuw. $i$ als auch das als Entsprechung von $a$ der anderen türk. Sprachen mitunter vorkommende tschuw. $\hat{\partial}$ aus $a$ entwickelt hat, s. a.a.O. 66, 80-81. V. G. JEGonov spricht von dem schon im Bolgarischen vor sich gegangenen Lautwandel $a>i(i)$, s. Sovremennyj čuvašskij literaturnyj jazyk I 162. Auch Is-chakov meint, das $\underset{i}{i}$ des Tuvinischen, Jakutischen und Tschuwaschischen habe sich als Resultat einer Art Reduktion aus a entwickelt, s. a.a.0. 66-69. Wie aus Räsänens Statistik a.a.O. 66 hervorgeht, entspricht tschuw. $i$ ebenso gut urtürk. kurzem wie auch langem $a$. Gleichfalls können tschuw. $\hat{\partial}$ und $ә$ sowohl aus urtürk. kur- 
zem als langem $\underline{i}$ (seltener aus kurzem und langem $a$ ) entstanden sein. Der Unterschied zwischen $\hat{\partial}(\partial)$ und $\boldsymbol{i}$ im Tschuwaschischen kann also nicht aus einem alten Quantitätswechsel erklärt werden, etwa so, dass â und a die kurze Quantitätsstufe eines gewissen Urlautes repräsentierten, $i$ wiederum seine lange Quantitätsstufe, oder umgekehrt.

Weil urtürk. $i$ im Tschuwaschischen zu $\hat{\partial}(\partial)$ wurde, ist offenbar zu folgern, dass dem jetzigen tschuw. $\underline{i}$ in irgendeiner früheren Phase ein Vokal vorangegangen sein muss, der q u a l it a t i v anders geartet - d.h. offener - als $\underline{i}$ (bzw. $\bar{\imath}$ ) war, der sich aber zugleich schon klar von a unterschied. Solche Ansichten hat man auch schon geäussert. In seinem bekannten Aufsatz "Zur Frage nach der Stellung des Tschuwassischen» sagt G.J. RAmstedT, indem er das Vorkommen von tschuw. $\hat{\partial}$ und $\underset{i}{i}$ als Entsprechungen von $a$ anderer türk. Sprachen behandelt, dass tschuw. $i$ sich aus einem älteren $e(\hat{\partial})$ entwickelt hat. Z.B. ist das ung. Wort tiló 'Hanfbreche, Schwinge' nach ihm aus einer alttschuw. Form *telâ $\gamma$ ( $>$ tschuw. tilla) entlehnt worden, s. JSFOu XXXVIII: 1 S. 7. Offensichtlich hat Ramstedt an eine Entwicklungsreihe ${ }^{*} a>$ ${ }^{*} e>^{*} \hat{a}$ (Reduktion) $>\underline{i}$ ("eine Verstärkung oder Verdeutlichung des â, vgl. a.a.O. 12) gedacht. Hier muss man aber fragen, ob nicht die Annahme einer Entwicklung $*_{a}>{ }^{*} e$ $>*_{i}>$ (sporadisch) $\hat{\sigma}$ natürlicher wäre. Diese Alternative bedentet, dass der Wandel $* \boldsymbol{e}>\boldsymbol{i}$ im allgemeinen erst dann stattgefunden hätte, als urtürk. $i$ schon zu $\hat{\partial}(\tilde{q})$ reduziert worden war; sporadisch hätte jedoch auch ein aus ${ }^{*} e$ entstandenes $i$ an der letztgenannten Vokalreduktion teilnehmen können. $\mathrm{Zu}$ beachten ist die Äusserung von G. Doerfer in UAJb 40 S. 244 , wo er zuerst sagt, das $\ddot{i}[=i]$ des Tuvinischen, Jakutischen und Tschuwaschischen gehe auf einen besonderen alttürk. Vokal $\ddot{e}[=e]$ zurück. Zunächst richtet er seine Aufmerksamkeit auf das Tschuwaschische, "wo (mit Ausnahme von $* \ddot{a}, * \ddot{a}>a$ und teilweise ${ }^{*} i>i$ ) sich als allgemeines Gesetz aufstellen lässt, dass sich die Vokale des Urtürkischen jeweils um eine Stufe 'gesenkt' haben, d.h. dass offene Vokale geschlossen wurden, geschlossene reduziert (allerdings gibt es viele Assimilationen, die das Bild verwir- 
ren, $k$ z.B. verschiebt die Vokale nach hinten, andere Laute wiederum nach vorn), also (offene Vokale) ${ }^{*} a>a>0>u$, ${ }^{*} \bar{e}>\ddot{i}, *_{o}>u ;{ }^{*} \ddot{a}>\dot{a}>a,{ }^{*} e>i,{ }^{*} \bar{o}^{1}>\ddot{u}$ (aber ${ }^{*} \bar{o}$ oft ăva - dagegen (geschlossene Vokale) $*_{\ddot{\imath}}>\check{e}^{2}, *^{*} u>\check{o}>\check{a}$; $*_{i}>\check{e}$ (aber eigenartigerweise kurzes $* i$ oft $>i$ ), * $\ddot{u}>\check{o}>\check{e}$.»

Hier wird nicht die Frage behandelt, ob tschuw. $i$ auf urtürk. ${ }^{*} a$ oder auf ein von diesem etymologisch getrenntes *e zurückgeht. Jedenfalls erscheint in den tschuw. Lehnwörtern des Wotjakischen als Entsprechung von tschuw. $i$ regelmässig ein offenerer Vokal als dieser, was darauf hinweist, dass während dieser Lehnberührungen auf tschuw. Seite an Stelle von $\underline{i}$ wirklich ein zunächst halbenger Vokal vorhanden war. Das fragliche Belegmaterial sieht wie folgt aus: wotjJ körś: vit-k., S kerś: vit-k. 'Abgabe, Steuer' < tschuw. (Ahlqv., ZoL.) xyrs', (in der Schriftsprache) xырс̧ӑ, xырą̧; wotjMU söu, K söl (l pro ü analogisch) 'gesund' < tschuw. sivâ, siv; wotjK söuliki, söllekk 'Gesundheit' < tschuw. sivlâ ; wotjG sesîr, J sösir, S sesir, $\mathrm{K}$ söser 'verstümmelt, verkrüppelt, Krüppel, Verstümmelter' < tschuw. (AHLQv.) $s y^{\prime} v$ zyr, (in der Schriftsprache) cbıcăp (<* sivâzâr); wotjG U sélịk, M S śelik, J sólik, MU śömîk, K śölek 'Sünde' < tschuw. sílâx $\chi$ vgl. YrJö Wichmann MSFOu XXI 30, 73-74, 92 $-93,99$. Die Vertretung des Vokals der ersten Silbe ist in den wotj. Dialekten sehr wechselnd: $e, \dot{o}, \ddot{o}, \ddot{o}, \ddot{g},($ vor $u) \ddot{n}(\mathrm{vgl}$. Wichmann, MSFOu XXXVI 17-18, J. BALASSA KSz. XVI 102, B. Munkácsi - D. R. Fuchs MSFOu 102 S. XXX, V. I. Lytkin Voprosy jazykoznanija 1968, Nr. 1 S. 86-88), aber alle diese dialektalen Varianten sind normale Vertreter eines urperm. ${ }^{*} \dot{d}$-Vokals, der ursprünglich lang war, vgl. Verf., UAJb 41 S. 249 . Aller Wahrscheinlichkeit nach war die Quantitätskorrelation ${ }^{*} \dot{a}-{ }^{*} \bar{g}$ im Wotjakischen zur Zeit der tschuw. Berührungen nicht mehr gültig. Vermutlich hatte sich $*^{*} \mathrm{zu} * \dot{o}$ verkürzt; frühurperm. kurzes $* \dot{o}$ dagegen war schon früher mit urperm. $*_{o}$ zusammengefallen, abgesehen

1 Offenbar Druckfehler statt *ö.

2 Auch hier vielleicht Druckfehler statt $\check{e}$, $\check{a}$, weil $\breve{a}(=\grave{j})$ im Tschuwaschischen als Vertreter von urtürk. * $\underset{i}{i}$ ungefähr ebenso häufig ist wie $\ddot{e}$. 
von den von Lytkin südwestlich genannten Dialekten, in denen es zu $u$ geworden war, vgl. Lytkin, ESA VII 226. Wenn die urperm. Quantitätsverhältnisse noch während der Aufnahme der tschuw. Elemente erhalten gewesen wären, so würden wir erwarten, dass der Vorgänger des jetzigen tschuw. $i$ zunächst durch kurzes $* \dot{a}$ und nicht durch langes $*_{\bar{o}}$ substituiert worden wäre. In diesem Falle hätten wir

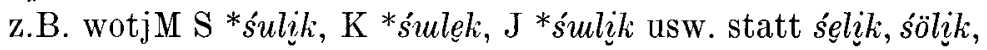
sólelk usw, aber eine solche Vokalvertretung ist in den Entsprechungen der tschuw. $i$-Wörter vollkommen unbekannt.

Es ist sicher, dass im Wotjakischen während der tschuw. Einwirkung neben $* \dot{a}$ noch ein etwas offenerer labialer Mittelvokal, urperm. ${ }^{*} \dot{o}$, existierte. Später veränderte sich dieser Laut in allen Dialekten des Wotjakischen zu o, vgl. Verf., FUF XXXI 289. Vor diesem Lautwandel wurden urperm. ${ }^{*_{o}}$ und $*_{0}$ im Wotjakischen zu $u$. In den Lehnwörtern dieser Sprache entspricht $* \dot{o}(>0)$ nie dem tschuw. $i$. Offenbar hat urtürk. ${ }^{*} i$ sich zu tschuw. $\hat{\partial}(\partial)$ durch Vermittlung eines engeren reduzierten Vokals ${ }^{*} \underset{\imath}{\imath}(=\hat{\imath})$ entwickelt, und eben der letztgenannte Laut muss in den Originalen der Lehnwörter des Wotjakischen vom Typus jîraך 'Grenze, Rain, Furche (auf dem Felde)' ( $<$ tschuw. jâran) angenommen werden; er ist also durch $i(\hat{\imath})$ substituiert worden, vgl. Wichmann, MSFOu XXI 30. Wenn man alle Umstände in Betracht zieht, so ergibt sich, dass es im Alttschuwaschischen, aus dem die tschuw. Lehnwörter des Wotjakischen stammen, als Vorgänger des $\underline{i}$ der jetzigen Sprache wahrscheinlich nicht ein ${ }^{*} e$, sondern einen etwas geschlosseneren Mittelvokal ${ }^{*} e$ gab, der folgerichtig durch den Laut substituiert wurde, der ihm in dem damaligen wotjakischen Vokalsystem am nächsten stand, nämlich durch ${ }^{*} \dot{a}$. Falls tschuwaschischerseits von ${ }^{*} e$ auszugehen wäre, so wäre ${ }^{*} \dot{o}(>0)$ das zu erwartende Pendant dieses Vokals im Wotjakischen.

Vergleichsweise betrachten wir wotjakische Entsprechungen auch anderer enger Vollvokale des jetzigen Tschuwaschischen. Dem tschuw. $i$ entspricht gewöhnlich $e$, wofür Wichmann, MSFOu XXI 32, acht Beispiele (z.B. be t'šei 'älterer Bruder' $<$ tschuw. piźźej; seti 'Zopfband' $<$ tschuw. síńa) 
anführt und zugleich berechtigterweise schliesst, dass "die Aufnahme der obengenannten wotjakischen Lehnwörter früher stattgefunden hat, als der Vokalübergang ${ }^{*} e>i$ im Tschuwassischen eingetreten war». In zwei Wörtern erscheint nach Wichmann das Verhältnis wotj. $i \sim$ tschuw. $i$ : wotj. itim, itịm usw. 'Dreschtenne' $<$ tschuw. dial. idem; wotj. sill 'Sturmwind' < tschuw. śil (< urtürk. *jel, s. Räsänen, Etym. Wb. d. Türksprachen 195); von diesen sagt er: "Da tschuw. $i$ auch in diesen Fällen wahrscheinlich aus urspr. $e$ entstanden ist, so muss für die entsprechenden wotjakischen Wörter gefolgert werden, dass sie erst nach dem Übergang

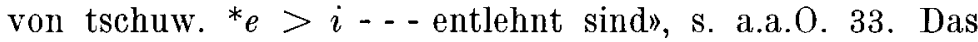
wotj. Wort sil hat aber auch eine seltene Nebenform sel, die offenbar archaistisch ist, vgl. T. E. Uotila JSFOu LII: 5 S. 2. Also scheint in diesem Wort die Schliessung eines $e$-Lautes zu $i$ erst auf dem wotjakischen Boden vor sich gegangen zu sein. Wenn man versucht, die Qualität des Vorgängers des tschuw. $i$ genauer zu bestimmen, so ist es logisch zu vermuten, dass sich nach Art der aus dem Urpermischen ererbten qualitativen Oppositionen $*_{\dot{0}}-*_{0}$ und $*_{0}-o$ (von dem letztgenannten Vokalpaar wird unten die Rede sein) im Wotjakischen zur Zeit der Aufnahme der tschuw. Lehnwörter auch die Opposition ${ }^{*} \ell-{ }^{*} e$ noch erhalten hatte. Jetzt hat sie sich so aufgehoben, dass in den einen Dialekten die $e^{-}$, in den anderen dagegen die $\ell$-Aussprache zur Herrschaft gelangt ist. Z.B. nach Balassa und Munkácsi-Fuchs ist den S-, J-, G- und K-Dialekten die erstere und den Sam.-, Uund P-Dialekten die letztere Variante eigen. Möglicherweise lag auch im Wandel ${ }^{*} e>i$ des Tschuwaschischen als Zwischenstufe ein *e vor, das zugleich in Originalen von Lehnwörtern des Wotjakischen hat begegnen können. Am besten erklärt sich das $i$ von wotj. siil eben so, dass in diesem Wort früher - und also vielleicht schon von dem Tschuwaschischen vererbt - das geschlossene $*_{\ell}$ vorkam, dessen sporadische Schliessung zu $i$ zwischen zwei mouillierten Konsonanten sehr verständlich gewesen wäre. (Zwar scheint das Wotjakische auch eine Nebenform *'sil mit nichtmouilliertem $l$ gekannt zu haben; aus ihr ist wohl syrjUd. siv [Fokos-Fuchs] 
'Wind' entlehnt worden, vgl. Uotila, a.a.O. 3.) Wenn im Lautverhältnis wotj. itìm, itîm 'Dreschtenne' $\sim$ tschuw. idem,

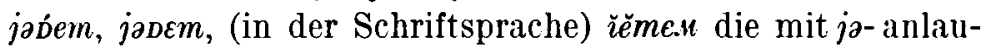
tenden tschuw. Formen das ältere Stadium repräsentieren, so kann man in dem tschuw. Wort eine schon vor seiner Entlehnung ins Wotjakische vor sich gegangene Entwicklung $* j a->i$ annehmen.

Die Entsprechungen von tschuw. $\ddot{u}$ sind nach Wichmann "sehr verschieden", s. a.a.0. 32. Es ist allerdings hervorzuheben, dass Wichmanns diesbezügliche Auslegungen eine gewisse Überprüfung erfordern. So ist unter den von ihm für sicher gehaltenen Fällen das wotjJ juśi, juśi, G S juśi 'Trauer, Kummer, Herzensbitterkeit; kläglich, traurig, jämmerlich (adv.)' offenbar kein ursprüngliches "ü-Wort», sondern aus irgendeiner hintervokalischen Form entlehnt. Für das betreffende tschuw. Wort jüźa rekonstruiert Räsänen eine urtürk. Urform *äcy, s. Etym. Wb. 4. Unter der Voraussetzung der Diphthongierung von urtürk. ${ }^{*} \bar{a}$ zu ${ }^{*} i a$ im Tschuwaschischen (vgl. Eino Karahka Studia Orientalia XIV: 11 S. 9-10) erhält man eine Entwicklungsreihe ${ }^{*} \vec{a}>{ }^{*} i a->{ }^{*} j a->{ }^{*} j \dot{a}->j 0^{-}>$ (dial.) $j u-\left(=\right.$ ZoL. juźe; vgl. z.B. ${ }^{*} \bar{a} s>{ }^{*}$ ias $>>$ jus 'Hermelin') $>$ (Palatalisierung als Folge des Zusammenwirkens von $j$ und nachfolgendem mouilliertem Sibilanten) jü-. Vollkommen berechtigt ist also die Annahme einer alttschuw. Form *jåżô, der wotj. juśi genau entspricht. Übrigens kann auf dieselbe Urform auch tscherW $j \grave{a} \cdot s \hat{\partial}$, tscherO JU $a_{j \grave{b}} \cdot s \otimes$,

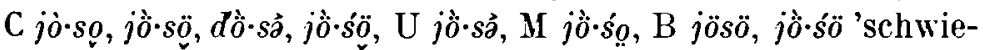
rig; Leiden' zurückgehen. Das inlautende $s$ des TscherW ist. aus ${ }^{*} s$ entstanden und setzt somit einen mouillierten Laut im tschuw. Original voraus; auch osttscheremissischerseits kann das dialektal rorkommende $s$ wenigstens stellenweise (z.B. im M-Dialekt) direkt von dem Tschuwaschischen herrühren. Ganz unwahrscheinlich bleibt somit die Annahme Wichmanns (s. FUF XVI Anz. 46), die Ausgangsform des tscher. Wortes sei tschuw. *jas, *jasâ (= uig. jas 'Schaden, Trauer' < urtürk. *jās, s. Räsänen, Etỵm. Wb. 191), voll dem man ung. gyász 'Trauer' hergeleitet hat. Unnötig ist es auch, nur tscherO jòsso, jồ śö als Entsprechung von tschuw. 
jüżo zu betrachten und tscherW $j \grave{a} \cdot s \hat{\partial}$ dagegen mit der Sippe *jās zu verknüpfen (auf solche Möglichkeit hat Räsänen, MSFOu XLVIII 138, verwiesen). Wäre dies richtig, so liesse sich im TscherW die Form *jà-šâ erwarten. Nicht erwartungsgemäss sind auch die Vokalverhältnisse zwischen wotj.

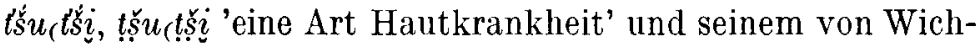

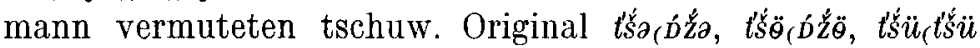
'Benennung einer Krankheit usw.'. Offenbar hat doch H. PAAsones recht, wenn er das wotj. Wort sowie auch tscher. čuči, čuču 'goldene Ader, Krebs' und mordM sućä, $\mathrm{E}$ ćuće 'eine gefährliche Hautkrankheit, Krebs' für Entlehnungen aus dem Tatarischen ansieht, vgl. tat. (PAss.) cöcö (misch. $\ddot{o}=$ kas. $\ddot{\theta}$ ) 'irgendeine Hautkrankheit', (RADL.) tö $\ddot{\theta}, s$. JSFOu XV: 2 S. 51, NyK XXXII 261. Der Vokal der ersten Silbe des tat. Wortes ist $\check{\ddot{o}}\left(<^{*} \ddot{\ddot{u}}\right.$, das in Normalfällen auf urtürk. ${ }^{*} \ddot{u}$ zurückgeht), dessen regelmässige Entsprechung in den älteren tat. Lehnwörtern des Wotjakischen eben $u$ ist, vgl. Wichmann, MSFOu XXXVI 38. Übrigens stammt

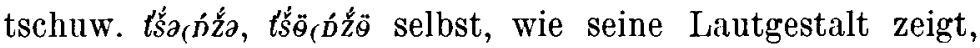
aus dem Tatarischen. (Ein anderes Wort ist tschuw. tšs-

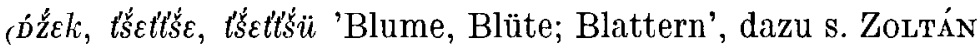
Gomвocz MSFOu XXX 59, Paasonen, JSFOu XV: 2 S. 51, N. Poppe UJb VII 163.)

Besser begründet ist es, folgende fünf Fälle als mögliche Repräsentanten der genannten Wortgruppe in Betracht zu ziehen. Ein sicheres, aus dem Tschuwaschischen entlehntes

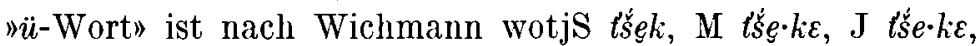
MU tšök 'Anruf bei dem Opfer für die Verstorbenen' (vgl. tschuw. t'śuk, t'śük, ZoL., AHLqv. ćuk 'Opfer'), bei dem die meisten wotj. Dialekte die ${ }^{*} \dot{q}$-Vertretung aufweisen. Das tschuw. Wort ist eine Entlehnung aus dem Tatarischen (vgl. tat. čüli 'ein Volksfeiertag', s. Räsänen, Etym. Wb. 113), und gegen Wichmanns Ansicht ist es wohl möglich, dass auch

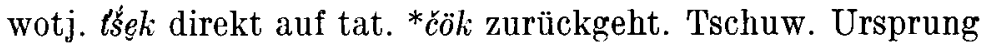
vermutet Wichmann zweifelnd für die Wörter wotjJ bodono, M bedono, bod'ono, U bedeno, MU bödono, K böd'ono, S bed'ono

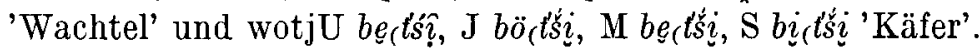

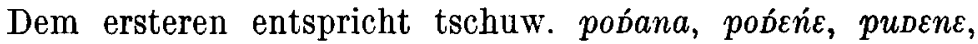


puDeñe, püDene id. Nach Munkácsi würde wotj. bod'ono usw. von tat. büdänä id. herrühren (NyK XVII 79), Wichmann aber begründet den tschuw. Ursprung des wotj. Wortes unter Hinweis darauf, dass der erwähnte tat. Vogelname nur ausserhalb des eigentlichen Wotjakengebiets vorkommt. Bei diesem türk. Worte ist von urtürk. ${ }^{*} \ddot{o}$ auszugehen, und falls wotj. bodono, bedono usw. ein tschuw. Lehnwort ist, so scheint in der gebenden Sprache zunächst die Vertretung $*_{\ddot{o}}$ ( $>$ wotjM $\mathrm{U} \mathrm{M} \mathrm{U} \mathrm{KS}{ }^{*} \dot{o}$ ), daneben aber auch noch ${ }^{*} \ddot{o}$ (> wotjJ $\mathrm{M}^{*} \dot{0}>\boldsymbol{o}$, wenn $\mathrm{J}$ M o seinen Grund nicht in der regressiven Assimilationswirkung seitens der nachfolgenden Labialvokale hat, auf welche Möglichkeit Wichmann hinweist) geherrscht zu haben. Der mutmasslichen urtürk. Form entspricht im Tschuwaschischen am besten die Variante pünée. Für das wotj. Wort bettś? usw. ist kein tschuw. Original bekannt, aber nach Wichmanns Auffassung könnte es "seines vokalischen Auslauts wegen” keine Entlehnung aus tat. bǚ̆äk, böz̆ek sein. Setzt man dies als richtig voraus, so hat das - jedenfalls aus dem Tatarischen übernommene - altschuw. Wort, von dem wotj. bett'ŝ herrührt, noch einen offeneren Vokal, zunächst $*_{a}^{*}$ gehabt, das im Wotjakischen durch ${ }^{*} \dot{a}$ ersetzt

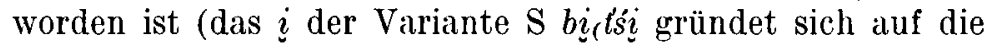
assimilierende Einwirkung des Vokals der zweiten Silbe). Wichmann behandelt in seiner Untersuchung nicht das wotj. Wort semolo 'Garbe; Schob, Schaub (Stroh od. Heu)', das eine offenkundige Entlehnung aus tschuw. śömel, śamel 'Heuhaıfen' ist, vgl. Munkácsi, NyK XVIII 121. (Merkwürdigerweise hält Wichmann, MSFOu XXXVI 39, es für ein Lehnwort aus dem Tatarischen, obgleich tat. čümälä in viele Dialekte des Wotjakischen in einer sich von semolo klar unterscheidenden Form, t'śumolo, übernommen wurde.) Auch dieses türk. Wort hat in der ersten Silbe ein urspr. * $\ddot{o}$, vgl. Jegorov, Etim. slov. čuv. jazyka 210. Wenn das den M-Dialekt repräsentierende, nur von B. GavriLov in seinem folkloristischen Werke von 1880 erwähnte und, von Munkácsi transkribiert, in NyK XVII 292 zu findende Wort semolo richtig geschrieben ist (Wichmann hält für möglich, dass hier ein Schreibfehler statt *sömolo vorliegt), so ist anzunehmen, dass das $e$ der ersten Silbe durch den palatalisierenden Einfluss 
von anlautendem $\dot{s}$ aus ${ }^{*} \boldsymbol{e}\left(<{ }^{*} \boldsymbol{j}\right)$ entstanden ist, wie sicherlich $e<e$ in $\mathrm{J} t \dot{s} e \cdot k \varepsilon(<t s \cdot k \varepsilon)$, s.o. Also hat es auch in diesem wotj. Worte früher wohl ein ${ }^{*} \dot{g}$ gegeben, das seinerseits ein ${ }^{*} \ddot{o}\left(<{ }^{*} \ddot{o}\right)$ im tschuw. Original voraussetzt. Im jetzigen Tschuwaschischen haben wir hier Angaben nur von der $\ddot{o}-$, $(>)$-Vertretung anstatt des zu erwartenden * $\ddot{u}$. Noch ist wotj. igi 'Uhu' zu untersuchen, das aus tschuw. $\ddot{u} G \partial, \ddot{u} \chi \partial, \ddot{u} \eta$ Gə id. entlehnt sein kann und in dem dann als Entsprechung von

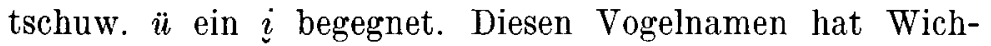
mann für ein Lehnwort sowohl tschuwaschischen (MSFOu XXI 32, 60) als auch tatarischen Ursprungs (MSFOu XXXVI 47) angesehen. In der ersten Silbe geht Räsänen zunächst vom urspr. ${ }^{*} \ddot{u}$ aus (Etym. Wb. 519), aber tschuw. $\ddot{u}$ ist als sein Vertreter ganz exzeptionell. Wenn es im Tschuwaschischen früher ein regelmässiges $* \ddot{u}$ gegeben hätte, so würde ihm, keinesfalls aber dem jetzigen Vollvokal $\ddot{u}$, wotj. $\underset{i}{\text { gut }}$ entsprechen. Die Hauptursache der lautlichen Unregelmässigkeit dieses Wortes, die mancherorts vorkommt, liegt ohne Zweifel in seinem onomatopoetischen Charakter.

So unsicher und vieldeutig das Material hinsichtlich der Entsprechungen von tschuw. $\ddot{u}$ auch ist, so können wir wohl doch konstatieren, dass es während der Lehnberührungen auf türkischer Seite ein $*_{o ̈}$ und zugleich vielleicht ein noch älteres $*_{\ddot{o}}$ gab. Das erstere wurde im Wotjakischen durch $*_{0} \dot{a}$ und das letztere - falls es wirklich existierte - durch ${ }^{*} \dot{o}$ substituiert. Später wurde ${ }^{*} \dot{g}$ in üblicher Weise zu $e\left({ }^{*} \dot{\partial}\right.$ zu $o$ ); in einigen Fällen hat sich ${ }^{*} e$ wahrscheinlich durch den Einfluss der Lautumgebung sporadisch zu $e$ bzw. $i$ verändert. Es ist ganz natürlich, dass die anzunehmenden alttschuw. Vokale $*_{\ddot{o}}$ und $*_{e}$ im Wotjakischen in $*_{\dot{q}}$ zusammenfielen; keiner von beiden hatte nämlich in der letztgenannten Sprache eine genaue lautliche Entsprechung.

Die Entwicklung von urtürk. ${ }^{*} o$ im Tschuwaschischen folgte nicht demselben Schema wie die der anderen urtürk. halbengen Vokale ${ }^{*} e,{ }^{*} e$ und ${ }^{*} \ddot{o} .{ }^{*} o$ ist hier vollkommen mit urtürk. ${ }^{*} a$ zusammengefallen. Die vom Verf. aufgeworfene Frage, ob dieser Zusammenfall so vor sich ging, dass *o zuerst mit $*_{a}$ verschmolz und dann ${ }^{*} a$ über die Zwischenstufe ${ }^{*} a$ 
zu o und im Anatri weiter zu $u(u)$ wurde (vgl. UAJb 41 S. 245), wäre wohl einer Prüfung wert. Auf eine solche Entwicklung können einige Vokalverhältnisse der tschuw. Lehnwörter im Tscheremissischen hindeuten, notwendig aber ist eine solche Annahme vielleicht nicht. Die tschuw. Lehnbestandteile des Mordwinischen und der permischen Sprachen erhellen dieses Problem nicht. Auf Grund des Mordwinischen und einiger syrj. Mundarten (SO, PO) sollte es theoretisch möglich sein, festzustellen, ob noch im Alttschuwaschischen ein Unterschied zwischen urtürk. ${ }^{*} a$ und ${ }^{*} o$ bestand; jedoch gibt es unter den wenigen tschuw. Lehnwörtern dieser Sprachen nur einige mit urtürk. ${ }^{*} a$ und - soweit wir wissen leider keines mit urtürk. ${ }^{*}$ o. Es fehlt also an Beweismaterial zur Unterstützung von Lytkins Vermutung, urtürk. $*_{o}$ sei im Permischen anfangs durch ${ }_{0}$, urtürk. ${ }^{*} a$ hingegen durch $*_{o}$ [ = Lytkin $*^{o}$ ] vertreten worden, s. Ist. vok. permsk. jazykov 108-111. Es ist offenbar, dass an sich die Opposition $o-o$, die sich im SO-Dialekt des Syrjänischen als solche und im Ostpermjakischen in etwas veränderter Form $(o>u)$ bewahrt hat, auch im Wotjakischen noch während der Berührungen mit dem Tschuwaschischen gültig war. Dafür spricht vor allem das tschuw. Lehnwort wotj. kulto, kulto, syrj. kolta, SO kolta 'Garbe', wovon S. 269 die Rede sein wird.

Tschuw. Lehnwörter des Syrjänischen, in denen der Vokal der ersten Silbe auf urtürk. ${ }^{*} a$ zurückgeht, sind wenigstens syrjPO koba 'Spindel' $\sim$ wotj. kubo id. < tschuw. *koba (das Wort ist im heutigen Tschuwaschischen nicht belegt) $\sim$ tat. kaba und syrjP PO torta 'Krücke od. Rechen zum Zusammenscharren des gedroschenen Getreides' wotj. turto 'Femerstange, Deichsel, Gabeldeichsel' < tschuw. torda, turpa tat. tarta, tärtä. Im Alttschuwaschischen war *å offensichtlich der Vertreter von urtürk. * $a$ der ersten Silbe (vgl. z.B. Verf., UAJb 41 S. 245 m.Lit.), und dieses * $a$ wurde im Permischen durch den nächststehenden Labialvokal, urperm. ${ }^{*} o$, substituiert. Wahrscheinlich gehört zur fraglichen Wortgruppe noch syrjP sor- 'Stief- (Anfangsteil gewisser Verwandtschaftsbenennungen)' $\sim$ wotj. sur- id. < tschuw. sor, sur $(\hat{\partial}) \sim$ tat. jarty, aber dies kann nicht sicher festgestellt werden, weil in 
den "Schlüsseldialekten» des Syrjänischen, die den Unterschied zwischen urperm. ${ }^{*} a$ und $*_{o}$ bewahrt haben, dieses Wort nicht vorkommt.

Interessante Fälle sind syrj. 'śarla 'Sichel' wotj. śurlo id. < tschuw. śorla, surla (etymologisch unklar) und syrj. adas 'Ackerbeet' $\sim$ wotj. udis id. < ? tschuw. (ein entsprechendes Wort ist im Tschuwaschischen nicht bekannt, hat aber vielleicht früher dort existiert, vgl. z.B. schor. adys 'Feldmass [1/18 Desjatine]' < urtürk. *atyz nach Räsänen, Etym. Wb. 31). Wichmann (MSFOu XXI 29) und Lytkin (Ist. vok. permsk. jazykov 108) haben gedacht, alttschuw. * $a$ der ersten Silbe hätte sich in den genannten syrj. Wörtern unverändert erhalten. Diese Wörter wären in diesem Falle die ältesten unter den tschuw. Lehnelementen des Permischen, weil man in ihnen keine Spur der Veränderung urtürk. ${ }^{*} a>$ tschuw. ${ }^{*} \boldsymbol{a}$ sieht, die sich in den übrigen Entlehnungen von * $a$-Wörtern widerspiegelt. Problematisch aber bliebe dabei der wotj. Lautwandel $a>u$, da urperm. $a$ sonst überall erhalten geblieben ist, z.B. syrj.-wotj. das 'zehn'. Eher wäre man geneigt zu denken, dass tschuw. ${ }^{*} \dot{a}$ im Wotjakischen normalerweise $>*_{o}>u$ wurde, aber im Syrjänischen infolge der regressiven Assimilierung (das $a$ der zweiten Silbe war vermutlich sogar der Träger des Hauptdrucks) sporadisch $>a$. Deskriptiv gesehen schliesst sich an beide letztgenannten Wörter noch das Wortpaar syrj. kan 'Zar' $\sim$ wotj. kun

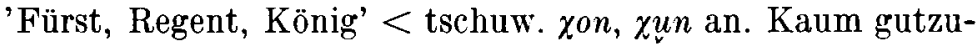
heissen ist die Annahme, syrj. kan sei eine von wotj. kun zu trennende Entlehnung aus dem Russischen, hat doch das Stammwort in beiden Sprachen sogar eine gemeinsame Ableitung: syrj. kanal-, wotj. kunjal- 'regieren, herrschen'. In Wirklichkeit gehört syrj. kan wotj. kun wohl zunächst mit den einsilbigen, vokalstämmigen Wortpaaren vom Typus syrj. $m a \sim$ wotj. $m u$ 'Honig', syrj. $s a \sim$ wotj. $s u$ 'Russ', syrj. $v a \sim$ wotj. $v u$ 'Wasser' zusammen. Es ist offensichtlich, dass es in ihnen während der urperm. Zeit kein $a$, sondern irgendeinen geschlosseneren Vokal gab. Möglicherweise entstand in solchen Wortstämmen ein kontrahierter gedehnter Vokal, der seine Länge noch ziemlich spät bewahrte. Die 
Situation wäre gewissermassen ähnlich wie im Tscheremissischen gewesen, wo der Vokal der betonten offenen Silbe im allgemeinen halblang, aber noch heutzutage z.B. in vielen von ÖDöN BEKF untersuchten west- und osttscher. Dialekten lang ist, wenn es sich um einsilbige Wörter handelt, z.B. $p \bar{u}$ 'Baum', 'gib!', liu 'Knochen', 'zehn', usw. Der anzunehmende späturperm. lange Kontraktionsvokal könnte ${ }^{*} \bar{o}$ bzw. ${ }^{*} \bar{o}$ gewesen sein, und im Wotjakischen wäre er, wie auch sonst qualitativ ähnliche Vokale, zu $u$ geworden, hätte sich aber im Syrjänischen zu a geöffnet. Vielleicht lässt sich denken, dass auch das perm. Wort kun $\sim$ kan früher einen exzeptionellen, langen Vokal enthielt. Türkischerseits weisen viele Sprachen in dem betreffenden Wort, alttürk. kayan, einen langen Kontraktionsvokal auf: kīn, $\chi \bar{a} n$. Darauf, dass auch im Alttschuwaschischen eine Form * $\chi \bar{a} n$ hat vorkommen können, die, sagen wir, als eine Art Emphatikum die Länge ihres Kontraktionsvokals erhalten hatte, scheint das von Beke aus den Uržumer, Malmyžer und Birsker Dialekten des Tscheremissischen aufgezeichnete, als zweiter Teil vieler mythologischer Namen vorkommende ōn 'Fürst' (vgl. Mari szövegek III 129-132, IV 26, 172) hinzudeuten. Dieses Wort ent-

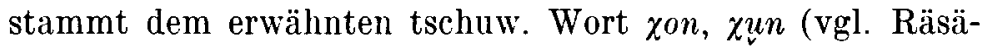
nen, MSFOu XLVIII 165), und sein langer Vokal in geschlossener Silbe widerspricht schroff den normalen Quantitätsverhältnissen. Das mutmassliche alttschuw. * $\chi \vec{a} n$ kann auf permischer Seite die Lautgestalt *kōn bzw. ${ }^{*} k \bar{o} n$ zu einer Zeit erhalten haben, als z.B. das Wort für 'Honig' noch in der Form ${ }^{*} m \bar{o}$ bzw. *mō vorlag.

Zur Ergänzung des oben Gesagten sei hinzugefügt, dass als Entsprechungen der urtürk. engen Vokale in den tschuw. Lehnwörtern des Wotjakischen ausschliesslich Vokale desselben Artikulationsgrades, d.h. $i, i, u(u)$, begegnen, vgl. des Näheren Verf., UAJb 41 S. 220. Nur ein Detail dieser Vokalgruppe soll hier in aller Kürze behandelt werden. Wie schon oben (S. 260) angeführt wurde, scheint sich in den südwestlichen Dialekten des Wotjakischen sehr früh der Mittelvokal $u$ entwickelt zu haben. Jedoch wurde innerhalb dieses Dialektgebiets das alttschuw. * ${ }_{\breve{u}}$ ( $>\breve{o ̈ ~ i m ~ N e u t s c h u w .) ~}$ 
nie durch $u$, sondern, wie auch in anderen Mundarten, durch $i$ ersetzt, obgleich theoretisch betrachtet ebenso gut auch $u$ hätte in Frage kommen können. Die Delabialisation von tschuw. * $\ddot{\ddot{u}}$ hat also auf gleiche Weise im ganzen wotj. Sprachgebiet stattgefunden. Dass tschuw. ${ }^{*} \ddot{u}$ zu $i$ und nicht zu $i$ wurde, beruht wohl darauf, dass die Artikulationsstelle von $\ddot{u}$ von Natur aus etwas weiter hinten liegt als die von $i$. Dagegen kommt z.B. im MU-Dialekt als Entsprechung von alttschuw. ${ }^{*} \check{u}(>\check{o}$ im Neutschuw.) sogar ziemlich oft $u$ vor, z.B. bus ( M bus) 'Nebel, Dampf, Wasserdampf' <

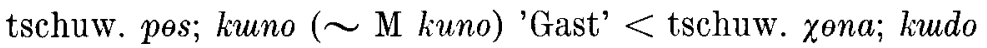
$(\sim \mathrm{M} k u d o)$ 'Brautwerber, Freiwerber' < tschuw. $\chi \theta D a$. Anders als im Wortschatz tschuw. Ursprungs wurde in älteren tat. Lehnwörtern des betr. Dialekts der palatale Labialvokal * $\ddot{u}(>\breve{o})$ durch $u$ substituiert, z.B. tus ( S tus) 'Antlitz' $<$ tat. tös. Hatte das alttschuw. ${ }^{*} \check{u}$ irgendeine palatale Nuance gleich dem entsprechenden Laut im Osmanischen, wo nach Räsänen (s. Zur Lautgesch. d. türk. Sprachen 88) "überhaupt» ein $u$ als Vertreter von urtürk. * $u$ begegnet? In älteren tat. Entlehnungen des Wotjakischen sind $*_{\breve{u}}(>\check{o})$ und $* \breve{u}(>\breve{o})$ der Originale in $u(u)$ zusammengefallen.

Doch gibt es ein paar wotj. Wörter, die als Pendant des $\check{o}$ des tschuw. Originals einen Labialvokal, nämlich $u(u)$, haben. Für wotjG J MU K kulto, M S kulto $\sim$ syrj. kolta, SO kolta 'Garbe' < tschuw, këlDE, köl'́e, (>) kalDE, kalDe hat Lytkin vermutet, dieses Wort sei ins Permische in der Form *költa $\left[=*_{k i \rho} l_{t} l a\right]$ aufgenommen worden, s. Ist. vok. permsk. jazykov 47 . Das späturperm. ${ }^{*} \dot{a}$ aber sollte sowohl im Wotjakischen als im Syrjänischen durch $e$ vertreten sein. Es ist glaubhaft, dass das tschuw. Wort permischerseits von Anfang an die im Syrjänischen erhaltene Lautform kolta bekam, m.a.W. scheint tschuw. * $\ddot{u}$ sowohl von anlautendem $k$ als auslautendem $a(<* \ddot{a}<$ tschuw. $\varepsilon$ ) beeinflusst worden zu sein: durch $k$ wurde es velarisiert und durch $a$ etwas offener. ${ }^{*} o$ veränderte sich im Wotjakischen in üblicher Weise $\mathrm{zu} u$, das in den südwestlichen Dialekten vor einem mouillierten Konsonanten in $u$ überging. Die letztgenannte Lautveränderung ist dort eine regelmässige Erscheinung, s. Wich- 
mann, MSFOu XXXVI 11. Das Wort wotj. kulto, syrj. kolta hat deswegen eine beachtliche lautgeschichtliche Bedeutung, weil es beweist, dass auch im Wotjakischen noch gegen Ende der permischen Sprachgemeinschaft ein von $o$ getrennter geschlossener $o$-Vokal vorhanden war. Ein anderes Wort, in dem $u$ dem tschuw. $\breve{~ e n t s p r i c h t, ~ i s t ~ w o t j . ~ b u k o ~}$

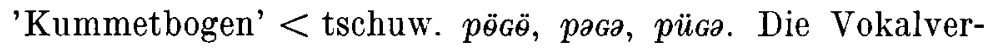
tretung im Tschuwaschischen ist schwankend; jedoch hat man offenbar von einem engen Vordervokal auszugehen, der sich im Wotjakischen hier wie im vorigen Worte neben $k$ sporadisch velarisiert hat. Dem Gedanken Wichmanns, dass schon die tschuw. Originale von wotj. kulto und buko hintervokalisch waren (MSFOu XXI 34), gewähren die sprachlichen Tatsachen wohl keine Stütze.

Von dem Vorgang urtürk. ${ }^{*} a>$ alttschuw. $*^{*} a>$ altperm. $*_{o}>$ wotj. $u$ haben wir reichlich Beispiele. In der Zeit der tschuw.-wotj. Berührungen hatte sich der entsprechende Vordervokal urtürk. ${ }^{*} \ddot{a}$ im Tschuwaschischen entweder unverändert bewahrt oder höchstens zum Mittelvokal ${ }^{*} \boldsymbol{a}$ entwickelt, und er wurde im Altpermischen, in dem es kein $\ddot{a}$ gab, durch a substituiert, s. Verf., UAJb 41 S. 245. Nur in einem Falle, wotj. ket's 'Ziege' $\sim$ syrj. kett's 'Hase' < tschuw.

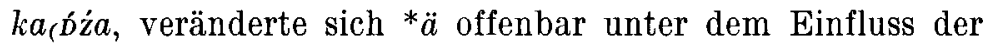
nachfolgenden mouillierten Affrikata $\mathrm{zu}{ }^{*} \varepsilon$ (dieses ist inlautend im Wotjakischen zu $e$, im Syrjänischen zu $e$ geworden). Tschuw. $\varepsilon$ hat im Wotjakischen eine zweifache Entsprechung, s. Wichmann, MSFOu XXI 31. Obgleich ein auslautendes $\varepsilon$ noch heute im Wotjakischen allgemein ist (z.B. gurte 'nach Hause', baśte 'er nimmt'), erscheint es nur in einem Teil der Fälle an der Stelle von tschuw. $\varepsilon$, z.B. wotjS U vème,

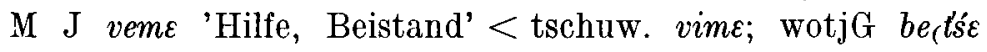
'Nachbar, Verwandter' < tschuw. pit'se = * pitt'se; wotjJ $\mathrm{S}$ $i s ́ k \varepsilon,(>)$ MU $s k \varepsilon$ 'in diesem Falle, wenn es so ist' < tschuw.

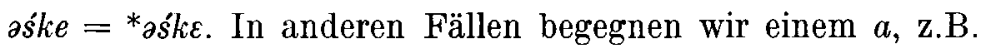
arna 'Woche' < tschuw. erńe (arna); śáśka, śaśka 'Blume, Blüte' < tschuw. śsśke. In der ersten Silbe scheint durchweg das letztgenannte Verhältnis zu herrschen, z.B. arak, araka 'Branntwein' < tschuw. $\varepsilon r \varepsilon \chi, \quad \varepsilon r \varepsilon \varepsilon \varepsilon ;$ sañik, saṇ̃̂k 'grosse 
Gabel' < tschuw. senək (sänak); arńa s.o.; śaśka, śaśka s.o. (Ausser in wotj. ket's $\sim \operatorname{syrj}$. ket's findet sich altperm. ${ }^{*} \varepsilon$ in der ersten Silbe wohl nur in einem tschuw. Lehnwort, nämlich wotjU J M S G ener, MU ener, (Musk.) K enär syrj. enịr 'Sattel' < tschuw. jancr, anär, joner; im altperm. Worte wurde tschuw. ${ }^{*}{ }_{i} \mathrm{zu}^{*} \varepsilon$ wahrscheinlich unter der assimilierenden Einwirkung des [hauptbetonten] $\varepsilon$ der zweiten Silbe.) Im Wortinnern in nichterster Silbe finden wir wieder eine doppelte Vertretung, einerseits $e$ bzw. (vor $i$ ) $\dot{e}$, das in einer komplementären Distribution zu auslautendem $\varepsilon$ steht (Munkácsi hat $\ddot{a}[=\varepsilon]$ auch inlautend notiert), anderseits a. Zu der ersteren Beispielgruppe gehören Beugungsformen der Wörter vom Typus veme, be t'ś (s.o.) und gewisse Ableitungen von ihnen (wie M bestśè 'älterer Bruder' < tschuw. pi(D́żej), ausserdem einige konsonantstämmige bzw. mehrsilbige Wörter: U J M G kallen, (Мusk.) K S kallän

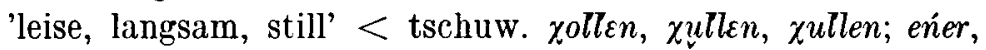
ener, (Muxk.) enär s.o.; keremet, keremet' 'Opferstelle, Opfer; böse Gottheit', falls dies aus tschuw. kiremet, kiremet, kiremet', keremet entlehnt und kein tat. Lehnwort ist. Die $a$-Vertretung herrscht im Wort arak, araka und in Beugungsformen der Wörter arńa und saśka (s.o.). Wir sehen also, dass die Lehnwörter des Wotjakischen in beträchtlichem Masse den Zusammenfall von tschuw. $\varepsilon(\sim e)$ mit tschuw. ${ }^{*} \ddot{a}$ aufweisen.

Oben ist das Bild, das Wichmann in seinem vor $67 \mathrm{Jah}-$ ren erschienenen Werk "Die tschuwassischen Lehnwörter in den permischen Sprachen" (MSFOu XXI) von der Vokalgeschichte der tschuw. Lehnwörter im Wotjakischen schuf, einigermassen überprüft worden unter Berücksichtigung dessen, was man heute über die Entwicklung des perm. und türk. Vokalismus im allgemeinen weiss. Dabei sollte man sich auf die Hauptlinien beschränken und gewisse unklare Einzelheiten übergehen. Wie schon oben erwähnt wurde, ist das Belegmaterial knapp und u.a. in der Hinsicht schwer zu beurteilen, dass man nicht immer entscheiden kann, ob irgendein Wort wirklich dem Tschuwaschischen oder aber dem Tatarischen entstammt. Dessen ungeachtet ist vor allem festzustellen, dass nicht nur das $i$ (vgl. Wichmann, a.a.0. 32), 
sondern überhaupt alle engen Vollvokale des jetzigen Tschuwaschischen im Alttschuwaschischen als Vorgänger etwas offenere Vokale hatten. Der Artikulationsgrad der genannten Vokale ist auffallend: nach Ausweis der Lehnwörter des Wotjakischen waren tschuwaschischerseits Laute wie ${ }^{*} e,{ }^{*} \ddot{o},{ }^{*} e$ vorhanden, die hinsichtlich ihrer eigenartigen Artikulationsöffnung ein Gegenstück zur urperm. Vokalreihe "zweiten Öffnungsgrades» bildeten. Die urtürk. engen Vokale hatten sich im Altschuwaschischen offenbar schon reduziert $\left({ }^{*} i>i\right.$, ${ }^{*} \underline{i}>\underline{i},{ }^{*} \ddot{u}>\check{\ddot{u}},{ }^{*} u>\check{u}$ ), und um die von ihnen hinterlassene leere Stelle unter den Vollvokalen durch Geschlossenwerden zu füllen, hatten ${ }^{*} e,{ }^{*} \ddot{o},{ }^{*} e$ sich schon in Bewegung gesetzt und lagen damals sozusagen auf der Mitte ihres Weges. Besonders soll aber betont werden, dass die Kategorie der engen Vollvokale im Neutschuwaschischen als Ganzes nicht auf diese Weise entstanden ist. Dank gewisser sporadischer "Vorläufer" hat sie schon früher anfangen können, sich zu gestalten. $\mathrm{Zu}$ beachten ist z.B. die von Doerfer erwähnte unregelmässige, aber oft vorkommende Erhaltung des urtürk. ${ }^{*} i$ vor der Reduktion, s. o. S. 259. Auch einige chronologische Schlussfolgerungen hinsichtlich des stufenweisen Zerfalls des urperm. Vokalsystems kann man auf Grund der tschuw. Lehnwörter ziehen.

Die Möglichkeit des Vorhandenseins der alttschuw. Vokale $*_{\ell}, *_{0}, *_{e} e$ ist auch dann zu erkennen, wenn man gewisse Vokalverhältnisse der tschuw. Lehnwörter im Tscheremissischen bewertet. U.a. kann der ziemlich oft auftretende dialektale Wechsel tscherW $i \sim 0$ e (z.B. W piträš $\sim 0$ petraš 'zuschliessen' < tschuw. pidar) natürlich so zu erklären sein, wie es Räsänen getan hat, dass nämlich die e-Vertretung von früheren Berührungen als die $i$-Vertretung herrührt (s. MSFOu XLVIII 99), aber man kann auch die Frage aufwerfen, ob in den Originalen von Wörtern dieses Typs ein alttschuw. ${ }^{*} \ell$ war, das einesteils durch $i$, andernteils durch $e$ ersetzt wurde. Dieselbe Frage entsteht anlässlich solcher Doppelheiten wie tscherW $\mathrm{O}$ tulaš $\sim \mathrm{O}$ tolaš 'Hanf brechen' < tschuw. tilla und W O uper $\sim \mathrm{O}$ oper 'Nachricht' < tschuw. $\chi i_{B}$ ar. Die allgemeinste Entsprechung von tschuw. $i \mathrm{im}$ Tscheremissischen ist jedenfalls $u$ (vgl. Verf., UAJb $41 \mathrm{~S} .217$ 
--218), aber das mitunter vorkommende tscherO o kann darauf hinweisen, dass auch hier, wie in den ins Wotjakische entlehnten Wörtern, ein offenerer Vokal als $\underline{i}$ Ausgangspunkt war, nämlich ein alttschuw. *e. Das von Beke in einigen tschuw. Lehnwörtern des Ceboksarer Dialektgebiets festgestellte $i$ (z.B. šißem 'zwei Fadenenden zusammenbinden' < tschuw. sip, s. FUF XXIII 83) erklärt sich durch Anpassung an die Aussprache des tschuw. Originals, welche Fähigkeit von einer ehemaligen ausgedehnten Zweisprachigkeit unter dortigen Tscheremissen zeugt. Was oben von den dialektalen Wechseln $i \sim e, u \sim o$ gesagt wurde, gilt mutatis mutandis auch von der zweifachen Vertretung $\ddot{u} \sim \ddot{o}$ als Entsprechung des aus urtürk. * $\ddot{o}$ entwickelten tschuw. $\ddot{u}$, d.h., hier kann wenigstens einigermassen ein alttschuw. *ö als Ausgangspunkt in Frage kommen.

Vielleicht aber fragt man: beruht nicht die Hypothese von einem alttschuw. ${ }^{*} e$ als Vorgänger des jetzigen tschuw. $i-$ die Verf. seinerseits für das interessanteste Ergebnis der Betrachtungen bezüglich des Vokalbestandes von tschuw. Lehnwörtern im Wotjakischen hält —, schon deswegen auf einer Fehldeutung, weil in den beträchtlich älteren tschuw. Lehnwörtern des Ungarischen als Entsprechung von tschuw. $i$ der enge Vokal $i$ erscheint (vgl. Gombocz, MSFOu XXX 144)? Wie früher erwähnt, wagte schon Ramstedt als Original von ung. tiló (vgl. tschuw. tịlâ) statt des von Gombocz rekon-

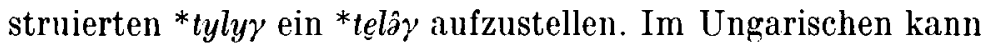
sehr gut eine Entwicklung $*_{e}>*_{i}(>i)$ vorausgesetzt werden, die unabhängig von der ähnlichen Entwicklung im Tschuwaschischen erfolgte. Die Forscher sind sich wohl darin einig, dass auch im bodenständigen Wortschatz des Ungarischen das $i(i)$ der ersten Silbe manchmal auf ein früheres ${ }^{*} i$ zurückgeht. Wie Verf. zuletzt in UAJb $41 \mathrm{~S} .98,248$ auseinandergesetzt hat, ist auch dieses Phonem wahrscheinlich aus einem offeneren Mittelvokal entstanden, der qualitativ und etymologisch dem ${ }^{*} \tilde{e}$ des Urwogulischen entsprach. Es ist ganz natürlich zu vermuten, dass beim Geschlossenwerden des urung. ${ }^{*} e \mathrm{zu}{ }^{*} i$ sowohl in fiugr. Wörtern als in tschuw. Lehnelementen auch ein ${ }^{*} e$ als $Z$ wischenphase hat existieren können.

ERKKI ITKONEN 